\title{
The efficacies of modified mechanical post conditioning on myocardial protection for patients undergoing coronary artery bypass grafting
}

Serkan Durdu ${ }^{1,2^{*}}$, Mustafa Sirlak ${ }^{1}$, Demir Cetintas ${ }^{1}$, Mustafa Bahadir Inan ${ }^{1}$, Sadik Eryllmaz ${ }^{1}$, Evren Ozcinar ${ }^{1}$, Levent Yazicioglu' ${ }^{1}$, Atilla Halil Elhan ${ }^{3}$, Ahmet Ruchan Akar ${ }^{1,2}$ and Adnan Uysalel ${ }^{1}$

\begin{abstract}
Background: Coronary artery bypass grafting (CABG) with cardioplegic cardiac arrest and cardiopulmonary bypass (CPB) is associated with myocardial injury. The aim of this study was to investigate whether a modified mechanical post-conditioning (MMPOC) technique has a myocardial protective effect by enhancing early metabolic recovery of the heart following revascularization.

Methods: A prospective, randomized trial was conducted at a single-center university hospital performing adult cardiac surgery. Seventy-nine adult patients undergoing first-time elective isolated multivessel coronary artery bypass grafting were prospectively randomized to MMPOC or control group. Anesthetic, cardiopulmonary bypass, myocardial protection, and surgical techniques were standardized. The post reperfusion cardiac indices, inotrope use and biochemical-electrocardiographic evidence of myocardial injury were recorded. The incidence of postoperative complications was recorded prospectively.
\end{abstract}

Results: Operative characteristics, including CPB and aortic cross-clamp time, were similar between the two groups $(p>0.05)$. The MMPOC group had lower troponin I and other cardiac biomarkers level post CPB and postoperatively, with greater improvement in cardiac indices $(p<0.001)$. MMPOC shortened post surgery hospitalization from $9.1 \pm 2.1$ to $7.5 \pm 1.6$ days $(p<0.001)$.

Conclusions: MMPOC technique promotes early metabolic recovery of the heart during elective $C A B G$, leading to better myocardial protection and functional recovery.

Keywords: Cardiopulmonary bypass, Myocardial protection, Ischemia-reperfusion injury, Coronary artery bypass grafting, Post-conditioning

\section{Background}

Reperfusion has the potential to cause additional reversible and irreversible damage to the myocardium, which is called reperfusion injury [1,2]. The existence of postconditioning (POC) is the newest evidence that has emerged to support the concept of reperfusion injury.

\footnotetext{
* Correspondence: serkandurdu@gmail.com

1 Department of Cardiovascular Surgery, Heart Center, Ankara University School of Medicine, Mamak Street, 06340, Dikimevi-Ankara, Turkey

${ }^{2}$ Stem Cell Institute, Ankara University, Ankara, Turkey

Full list of author information is available at the end of the article
}

The term post conditioning refers to the phenomenon in which multiple brief periods of reperfusion interspersed with brief periods of ischemia (10-60 s) result in a reduction in infarct size [3,4]. Generally, three cycles of ischemia/reperfusion are required to produce a maximal POC effect, although four and six cycles have been shown to be effective by some investigators $[3,4]$. However, it is the interval of reperfusion and ischemia that is the most critical factor in determining how efficacious POC will be. POC protocols shown to be maximally effective at reducing infarct size range from 10 to $60 \mathrm{~s}$ depending on the specific species being studied $[3,4]$.

\section{Biomed Central}


The aim of our study was to determine the efficacy of a modified type of mechanical post-conditioning (MMPOC) in patients undergoing elective coronary revascularization, with specific attention to biochemical markers of ischemic injury and post-surgical recovery of the patients and to show whether there is room for protection by post-conditioning amongst all the other cardioprotective factors.

\section{Methods}

\section{Patients and protocol}

This study was approved by the Institutional Review Board of the University of Ankara and consisted of 79 patients undergoing elective primary coronary revascularization with $\geq 99 \%$ stenosis of the left anterior descending (LAD) artery. So as to include patients with relatively large volumes of at-risk myocardium, we limited our analysis to those patients exhibiting proximal occlusion of the LAD. Patients undergoing valve replacement, combined valve replacement/coronary revascularization, or preoperative coronary revascularization was excluded from the study. Informed consent was obtained from all patients before enrollment.

\section{Randomisation}

Patients were allocated to the MMPOC group or the study group using a computer-generated randomization code. Participants were randomly assigned in a 1:1 ratio. The study was open label and the primary investigator, who was not the treating physician and nurses informed the participants about their allocated treatment. Data for primary outcomes were assessed by use of a computer.

\section{Data collection and definitions}

Baseline, procedural, and follow-up data were stored prospectively in a database located at the University of Ankara. Patients' preoperative risk factors were recorded and EuroSCOREs were calculated for each patient. Patients' preoperative characteristics were recorded including age, sex, size, preexisting medical conditions, preoperative medications, preoperative ejection fractions. Intraoperative variables of which number of coronary bypass grafts, duration of cardiopulmonary bypass (CPB), duration of aortic cross-clamp, requirement for inotropic drugs, and/or intra-aortic balloon support, and blood product use were included. Postoperative data comprised myocardial infarction, cardiac tamponade, reoperation for occlusion or other causes, requirement of intra-aortic balloon pump support, neurologic complications, renal dysfunction, chest tube drainage during the first 24 postoperative hours, total chest tube drainage, the length of mechanical ventilator support, pneumonia, multiorgan failure, gastrointestinal complications, sepsis, coma, the length of intensive care unit (ICU) stay, and readmission within 90 days after surgery.

Adverse events were defined as death, perioperative myocardial infarction, stroke, re-exploration due to bleeding, respiratory insufficiency, and renal failure. Perioperative myocardial infarction (MI) was defined as either new Q waves or ischemic ST segment changes with concomitant elevations of creatine kinase isoenzyme (CK-MB) $>5$ times the upper limit of the reference range or a CK-MB to total creatine kinase (CK) ratio > $10 \%$ occurring within 48 hours after surgery or troponin I $(\mathrm{TnI})>1 \mathrm{ng} / \mathrm{mL}$. Renal dysfunction was defined as rise of serum creatinine above $2.5 \mathrm{mg} / \mathrm{dL}$ and/or a need for hemodialysis. The surgical team examined all patients about 4-6 weeks after discharge and annually thereafter for two years.

\section{Anesthetic and surgical considerations}

Anesthesia was maintained with isoflurane. Hypertension was treated by increasing the concentration of isoflurane or by the administration of nitroglycerin if increasing the depth of anesthesia was ineffective. Hypotension was corrected using volume replacement or phenylephrine, as clinically indicated. An additional dose of $5 \mathrm{mg}$ of midazolam was provided during rewarming from $\mathrm{CPB}$. Inotropic agents (dobutamine $5 \mu \mathrm{g} / \mathrm{kg} / \mathrm{min}$ ) were initiated for a cardiac indices (CI) $<2.0 \mathrm{~L} / \mathrm{min} / \mathrm{m}^{2}$ after separation from CPB. At sternal closure, an infusion of propofol was started (25-75 $\mu \mathrm{g} /$ $\mathrm{kg} / \mathrm{min}$ ), and the isoflurane was discontinued. Propofol sedation was continued in the ICU until weaning of ventilatory support was initiated.

All patients had coronary artery bypass grafting (CABG) with the use of $\mathrm{CPB}$, which was conducted with a roller pump and a membrane oxygenator primed with a solution consisting of $1 \mathrm{~L}$ of Ringer's lactate, $5000 \mathrm{IU}$ of heparin, $750 \mathrm{~mL}$ of Pentaspan (DuPont Pharmaceuticals Co, Newark, DE), and $44 \mathrm{mEq}$ of bicarbonate. During $\mathrm{CPB}$, pump flow was set at 2.4 times the body surface area, and mean arterial pressure maintained between 50 and $60 \mathrm{~mm} \mathrm{Hg}$. The temperature was allowed to drift with active rewarming at the end of CPB. Cardioplegia solution (Plegisol, Hospira,Inc, Lake Forest, IL), which was used at the discretion of the cardiac surgeon, was free of glucose and consisted of high-dose $(100 \mathrm{mEq} / \mathrm{L})$ and low-dose $(40 \mathrm{mEq} / \mathrm{L})$ potassium. Cardioplegia was given in an anterograde fashion with blood in a ratio of 1:4. Blood cardioplegia was also administered with each successive distal vein graft anastomosis. The proximal vein graft anastomosis were performed after the distal ones under tangential aortic clamp. The left internal thoracic artery was used in all cases to bypass the LAD, while other coronary arteries received saphenous vein grafts. In patients with severe aortic 
disease where intermittent cross-clamping may be harmful, we used the MMPOC technique immediately after completion of the proximal anastomosis and weaning off $\mathrm{CPB}$, in the MMPOC group we applied bulldog clamps to the grafts anatomized to the coronary arteries as in a similar manner and duration explained by Zhao et al before 2 [4]. Our MMPOC technique consisted of applying three 30-sec alternate episodes of occlusion with bulldog clamps and releasing of the bypassed grafts providing reperfusion. This method was applied immediately after $\mathrm{CPB}$ and in this way uncontrolled reperfusion was disallowed in the study group (Figure 1). This method was applied immediately after CPB and in this way uncontrolled reperfusion wasn't permitted in the study group.

Functional recovery and postoperative cardiac biomarkers The CI, hemodynamic parameters and cardiac biomarkers were evaluated in 9 different time points (immediately after the induction of anesthesia; following completion of the proximal anastomosis; immediately off $\mathrm{CPB}$; immediately after MMPOC; 2, 6, 12, 24, and 48 hours-post MMPOC). The CI was calculated to determine the change in the $\mathrm{CI}$ as an indicator of functional recovery. Central venous pressure (CVP), mean pulmonary artery pressure (MPAP) and pulmonary capillary wedge pressure (PCWP) measurements were compared at the same time points to ensure similar preload filling status.

The baseline values of serum CK-MB, and TnI concentration were taken as described above. All the TnI samples were stored deep-frozen for quantitative analysis. TnI was analyzed using an AxSYM TnI Reagent Kit as a micro particle enzyme immunoassay method and an AxZYM analyzer (Abbott Laboratories, Abbot Park, IL). The upper reference limit was $0.50 \mathrm{~g} / \mathrm{L}$, and the cutoff limit for diagnosing acute myocardial infarction was $1.00[\mathrm{~g} / \mathrm{L}]$.

\section{Sample size and randomisation}

A repeated measures design has MMPOC and control groups of 39 and 40 subjects respectively for a total of 79 patients. Each subject is measured 6 times. The between-subject standard deviation is 0.41 and the within-subject standard deviation is 1.00 . This design achieves $96 \%$ power when an F test is used to test the group-factor at a 5\% significance level and the actual standard deviation among the appropriate means is 0.17 (an effect size of 0.42 ), achieves $91 \%$ power when an $\mathrm{F}$ test is used to test the time-factor at a $5 \%$ significance level and the actual standard deviation among the appropriate means is 0.19 (an effect size of 0.19), and achieves $83 \%$ power when an $\mathrm{F}$ test is used to test the (group X time) interaction at a 5\% significance level and the actual standard deviation among the appropriate means is 0.17 (an effect size of 0.17) [5].

\section{Statistical analysis}

Nominal variables were assessed by Chi square test or Fisher's Exact test, where applicable. Continuous and ordinal variables were evaluated between two groups by Student's $t$ test and Mann-Whitney $U$ test, respectively. Two-way analysis of variance (ANOVA) for repeated measures was used to assess the main effect of time as within-factor and post conditioning versus control as grouping factor and interactions between them (group $\mathrm{X}$ time). If a significant effect was detected, pair wise comparisons were evaluated using the Bonferroni test. A $p$ value less than 0.05 was considered significant. All

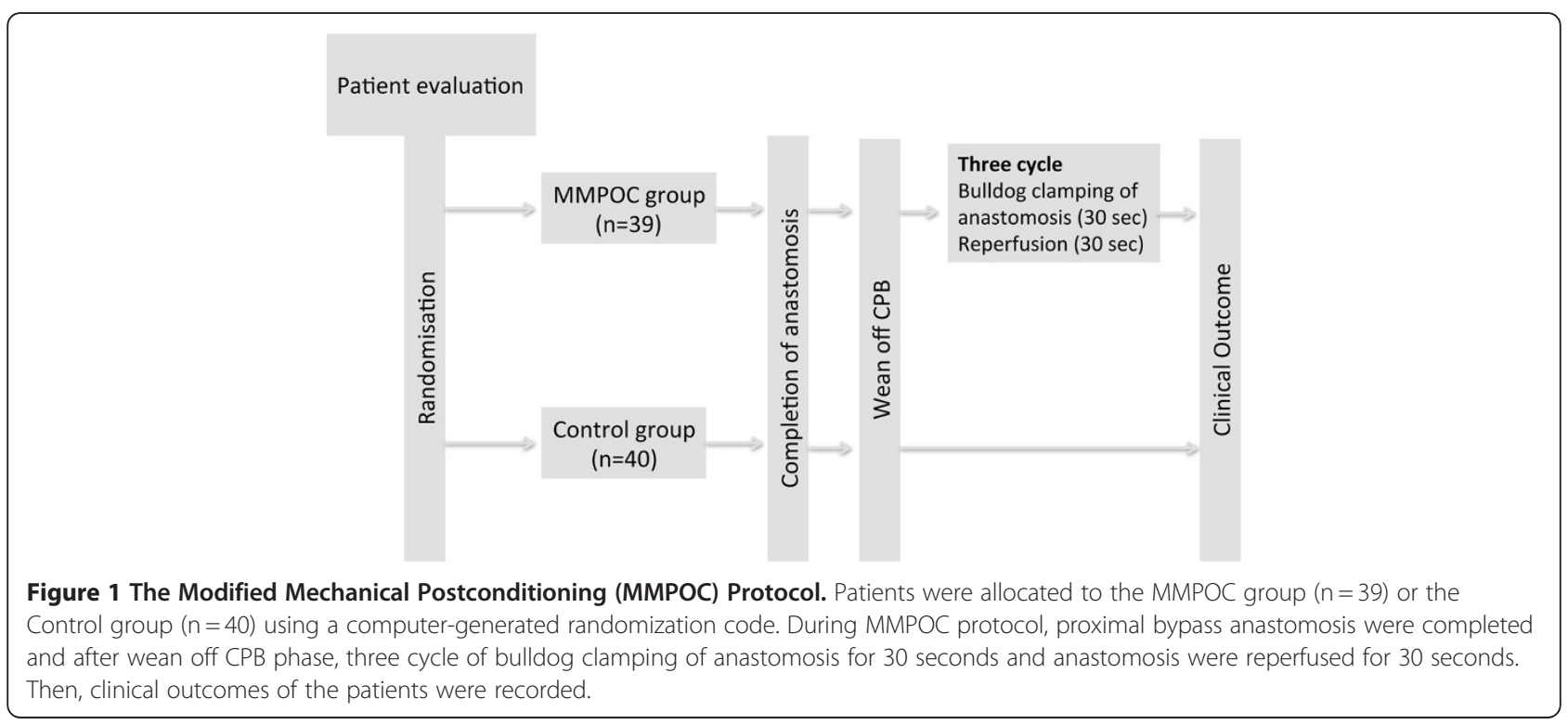


analyses were performed using SPSS $^{\circledR}$ Statistics 18.0 (SPSS Inc, Chicago, Illinois, USA).

\section{Results}

The patient characteristics as well as preoperative cardiac biomarkers and hemodynamic variables were similar within each study group ( $>>0.05)$ (Table 1 and Figure $2 \mathrm{~A}, \mathrm{~B}, \mathrm{C})$. The kinetics of cardiac biomarkers followed almost the same route and remained lower in the study group throughout the study period. But statistically significant differences occurred in different time points. CK and CK-MB remained lower in the study group from the $6^{\text {th }}$ and $12^{\text {th }}$ post-conditioning hours till the end of the study respectively in the MMPOC group $(p<0.001)$. Although TnI levels remained lower in the study group, the values reached statistical difference after the $24^{\text {th }}$ hours $(\mathrm{p}<0.001)$ (Figure $\left.2 \mathrm{~A}, \mathrm{~B}, \mathrm{C}\right)$.

\section{Intraoperative characteristics}

The CPB time was not statistically different between the groups $(p=0.062)$ nor was the aortic cross-clamp time $(p=0.082)$. The mean number of grafts was similar between the groups $(\mathrm{p}=0.974)$. The operative time was shorter in the MMPOC group (Table 2), however it was statistically insignificant $(\mathrm{p}=0.619)$. This period also include the post conditioning time. CI were comparable between the two groups in the first period $\left(2.01 \pm 0.10 \mathrm{~L} / \mathrm{min} / \mathrm{m}^{2}\right.$ in MMPOC group versus $1.99 \pm 0.09 \mathrm{~L} / \mathrm{min} / \mathrm{m}^{2}$ in control group, $\mathrm{p}=0.362$ ). Under the similar preload filling status, patients in MMPOC group had higher postconditioning $\mathrm{CI}$ in all time points $(\mathrm{p}<0.001)$. The actual values of PCWP, MPAP and CVP remained similar between the two groups in all time points (p>0.05) (Figure $3 \mathrm{~A}, \mathrm{~B}, \mathrm{C}, \mathrm{D})$.

Table 1 Patient Characteristics

\begin{tabular}{|c|c|c|c|c|}
\hline & & MMPOC $(n, 39)$ & Control $(n, 40)$ & P Value \\
\hline \multirow[t]{2}{*}{ Age $(Y r)$} & Mean \pm SD & $61.6 \pm 8.1$ & $62.4 \pm 7.1$ & 0.833 \\
\hline & Median (Min-Max) & $62(45-73)$ & $63(48-77)$ & \\
\hline \multirow[t]{2}{*}{ NHYA } & Mean \pm SD & $1.87 \pm 0.76$ & $1.72 \pm 0.67$ & 0.431 \\
\hline & Median (Min-Max) & $2(1-4)$ & $2(1-3)$ & \\
\hline \multirow[t]{2}{*}{ LVEF (\%) } & Mean \pm SD & $47.6 \pm 5.5$ & $46.1 \pm 5.7$ & 0.185 \\
\hline & Median (Min-Max) & $48(38-57)$ & $44.5(39-57)$ & \\
\hline \multirow[t]{2}{*}{ EuroSCORE } & Mean \pm SD & $3.05 \pm 1.37$ & $2.67 \pm 1.42$ & 0.193 \\
\hline & Median (Min-Max) & $3(1-6)$ & $2(1-6)$ & \\
\hline LMCA disease & n (\%) & $3(7.7)$ & $2(5.0)$ & 0.675 \\
\hline History of Hypertension & n (\%) & $15(38.5)$ & $17(42.5)$ & 0.715 \\
\hline Diabetes Mellitus & n (\%) & $15(38.5)$ & $17(42.5 \%)$ & 0.715 \\
\hline \multirow[t]{2}{*}{$\mathrm{Hb}(\mathrm{g} / \mathrm{dl})$} & Mean \pm SD & $12.1 \pm 0.46$ & $12.3 \pm 0.57$ & 0.358 \\
\hline & Median (Min-Max) & $12.3(10.6-13.1)$ & $12.3(11.8-14.5)$ & \\
\hline \multirow[t]{2}{*}{ Glucose (mg/dl) } & Mean \pm SD & $101.4 \pm 14.8$ & $98 \pm 12.1$ & 0.376 \\
\hline & Median (Min-Max) & $98(75-131)$ & $95(75-125)$ & \\
\hline \multirow[t]{2}{*}{ Creatinine (mg/dl) } & Mean \pm SD & $1.13 \pm 0.19$ & $1.15 \pm 0.15$ & 0.484 \\
\hline & Median (Min-Max) & $1.1(0.8-1.8)$ & $1.2(0.9-1.4)$ & \\
\hline \multirow[t]{2}{*}{ BUN (mg/dl) } & Mean \pm SD & $25.6 \pm 4.9$ & $26.5 \pm 5.1$ & 0.421 \\
\hline & Median (Min-Max) & $25(14-36)$ & $25(20-34)$ & \\
\hline \multirow[t]{2}{*}{ Protein (mg/dl) } & Mean \pm SD & $6.60 \pm 0.19$ & $6.61 \pm 0.22$ & 0.841 \\
\hline & Median (Min-Max) & $6.6(6.1-7.0)$ & $6.7(6.2-7.2)$ & \\
\hline \multirow[t]{2}{*}{ Albumin (mg/dl) } & Mean \pm SD & $3.50 \pm 0.20$ & $3.46 \pm 0.13$ & 0.277 \\
\hline & Median (Min-Max) & $3.5(3.0-3.9)$ & $3.4(3.3-3.8)$ & \\
\hline \multirow[t]{2}{*}{ INR } & Mean \pm SD & $1.05 \pm 0.07$ & $1.03 \pm 0.07$ & 0.450 \\
\hline & Median (Min-Max) & $1.02(0.9-1.3)$ & $1.02(0.8-1.2)$ & \\
\hline
\end{tabular}

$S D=$ standard deviation, Min = minimum, Max: maximum, MMPOC = modified mechanical post-conditioning NYHA, New York Heart Association, $L V E F=$ left ventricular ejection fraction, $L M C A=$ left main coronary artery, $H b=$ hemoglobin, $B U N$ : blood urea nitrogen, INR: international normalized ratio. Nominal variables were assessed by Chi square test or Fisher's Exact test, where applicable. Continuous and ordinal variables were evaluated between two groups by Student's $t$ test and Mann-Whitney $U$ test, respectively. 


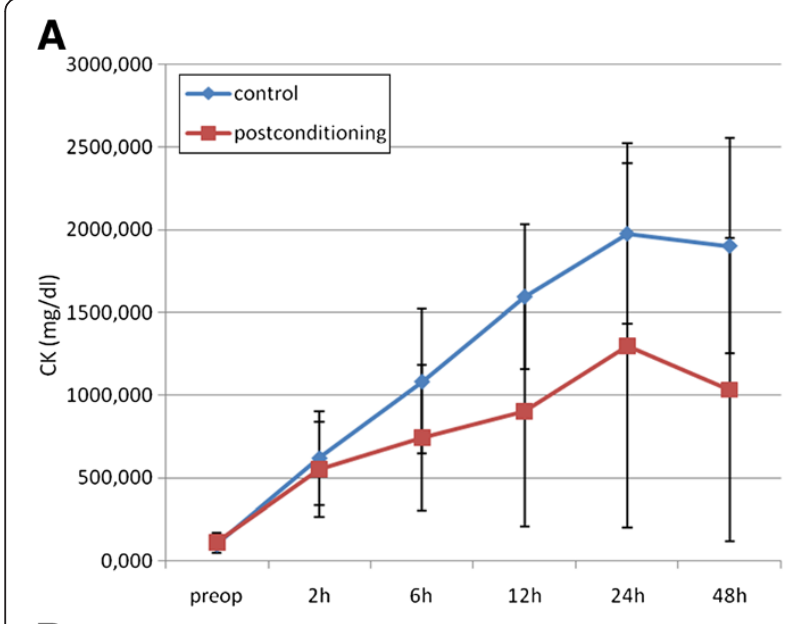

B

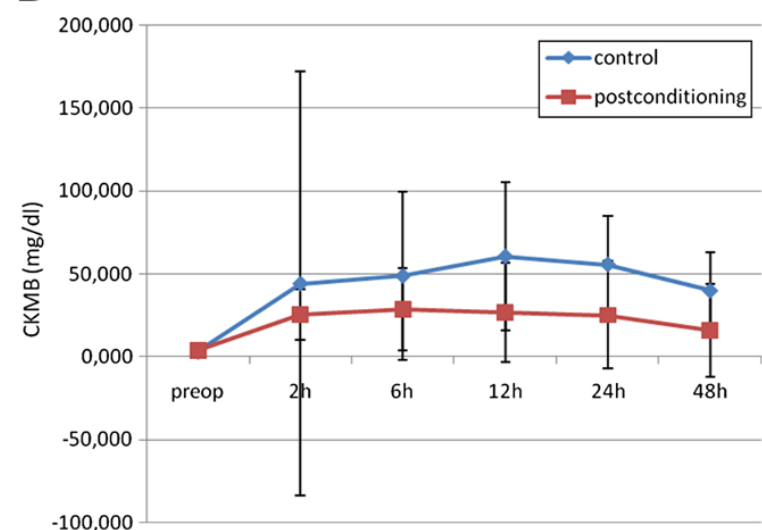

C

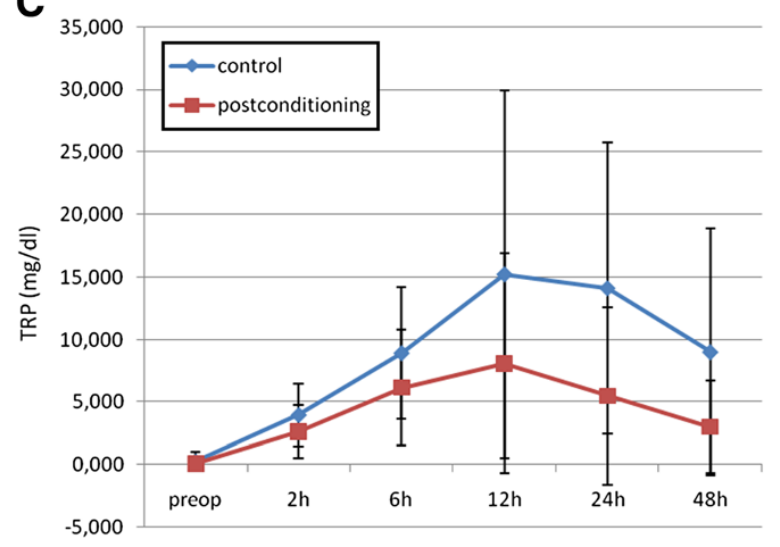

Postoperative characteristics

The median length of intubation was similar between the groups $(p=0.818)$. The MMPOC group patients had lower length of stay in the ICU and in the hospital compared to the control group $(\mathrm{p}<0.001)$. Importantly, use of MMPOC shortened postoperative hospitalization by more than one day. Postoperative complications were similar in both groups. There were three and five
Figure 2 The Kinetics of Cardiac Biomarkers. A; Time Course of Creatine Kinase (CK). Preoperative CK values were statistically insignificant for the control and postconditioning groups. Postoperative 2 hour point, CK values were similar. After 6 hour, CK levels for the MMPOC group and Control group began to depart and this significant difference continued to the 12 hour, 24 hour and 48 hour after the procedure. B; Time Course of Creatine Kinase Myocard-Brain (CK-MB). Preoperative CK-MB values were statistically insignificant for the control and postconditioning groups. Postoperative 2 hour point, CK-MB values for the two groups were began to differentiated statistically. CK-MB levels for the MMPOC and Control group were statistically significant for the 6 hour, 12 hour, 24 hour, and 48 hour time points. C; Time Course of Troponin-I (Tnl). Preoperative $\mathrm{Tnl}$ values were statistically insignificant for the control and postconditioning groups. Postoperative 2 hour point, Tnl values were similar. After 6 hour, Tnl levels for the MMPOC group and Control group began to depart and this significant difference continued to the 12 hour, 24 hour and 48 hour after the procedure. Blue line: Control, Red line: MMPOC.

perioperative myocardial infarctions in MMPOC and control groups respectively $(\mathrm{p}=0.712)$. While two hospital deaths were seen in control group, none of the patients died in MMPOC group $(\mathrm{p}=0.499)$. Wound infection rates, amount of bleeding and postoperative atrial fibrillation incidences were similar between the groups as shown in Table 3. The improvement of left ventricular function in the MMPOC group tended to decrease requirements for pharmacologic inotropic support $(\mathrm{p}=0.004)$.

\section{Discussion}

This study demonstrates that MMPOC is associated with increased CI and less cardiomyocyte injury. These findings are important because previous studies have demonstrated that low cardiac output, the need for inotropic support, and biochemical evidence of myocyte injury are important risk factors for prolonged ventilation, ICU stay, and early mortality after CABG [6]. The requirements for pharmacologic inotropic support during the post bypass period reached to statistical significance between the groups. Importantly, use of MMPOC shortened postoperative hospitalization by more than one day.

In our study, we hypothesized that post conditioning would provide protection against myocardial ischemiareperfusion in adult patients undergoing $\mathrm{CPB}$ for $\mathrm{CABG}$ and therefore conducted a controlled trial to evaluate the effect of post conditioning on myocardial protection. We applied a new method for the similar hypothesis set up in the other post-conditioning studies [7-11]. Our study setting was different compared to the familiar studies especially for the initiation of the postconditioning protocols. Our method started in the time of weaning from $\mathrm{CPB}$ while all the remaining studies started after aortic declamping. Although larger 
Table 2 Intraoperative characteristics

\begin{tabular}{llccc}
\hline & & MMPOC $(\mathbf{n}=\mathbf{3 9})$ & Control $(\mathbf{n}=\mathbf{4 0})$ & $\boldsymbol{P}$ value \\
\hline Operation Time (min) & Mean \pm SD & $154.3 \pm 17.0$ & $158.3 \pm 22.6$ & 0.619 \\
CPB Time (min) & Mean \pm SD & $74.1 \pm 27.3$ & $81.7 \pm 17.0$ & 0.062 \\
Cross Clamp Time (min) & Mean \pm SD & $41.2 \pm 14.9$ & $44.7 \pm 8.9$ & 0.082 \\
IABP & $\mathrm{n}(\%)$ & $0(0)$ & $2(5)$ & 0.241 \\
No. of graft/patient & & $2.92 \pm 0.83$ & $2.87 \pm 0.64$ & 0.974 \\
\hline
\end{tabular}

$M M P O C=$ modified mechanical post-conditioning, $S D=$ standard deviation, $C P B$, cardio-pulmonary bypass, IABP, intra aortic balloon pump.

Nominal variables were assessed by Chi square test or Fisher's Exact test, where applicable. Continuous and ordinal variables were evaluated between two groups by Student's $t$ test and Mann-Whitney $U$ test, respectively.

controlled clinical trials are needed to determine the effect of ischemic post conditioning on the reduction of myocardial necrosis in cardiac surgery, the results of the study conducted by Luo et al. Provided support for ischemic post conditioning as an adjunct to cardioplegia in reduction of myocardial necrosis in adult cardiac surgery [7]. Parallel to the aforementioned studies and statements, our study investigated the
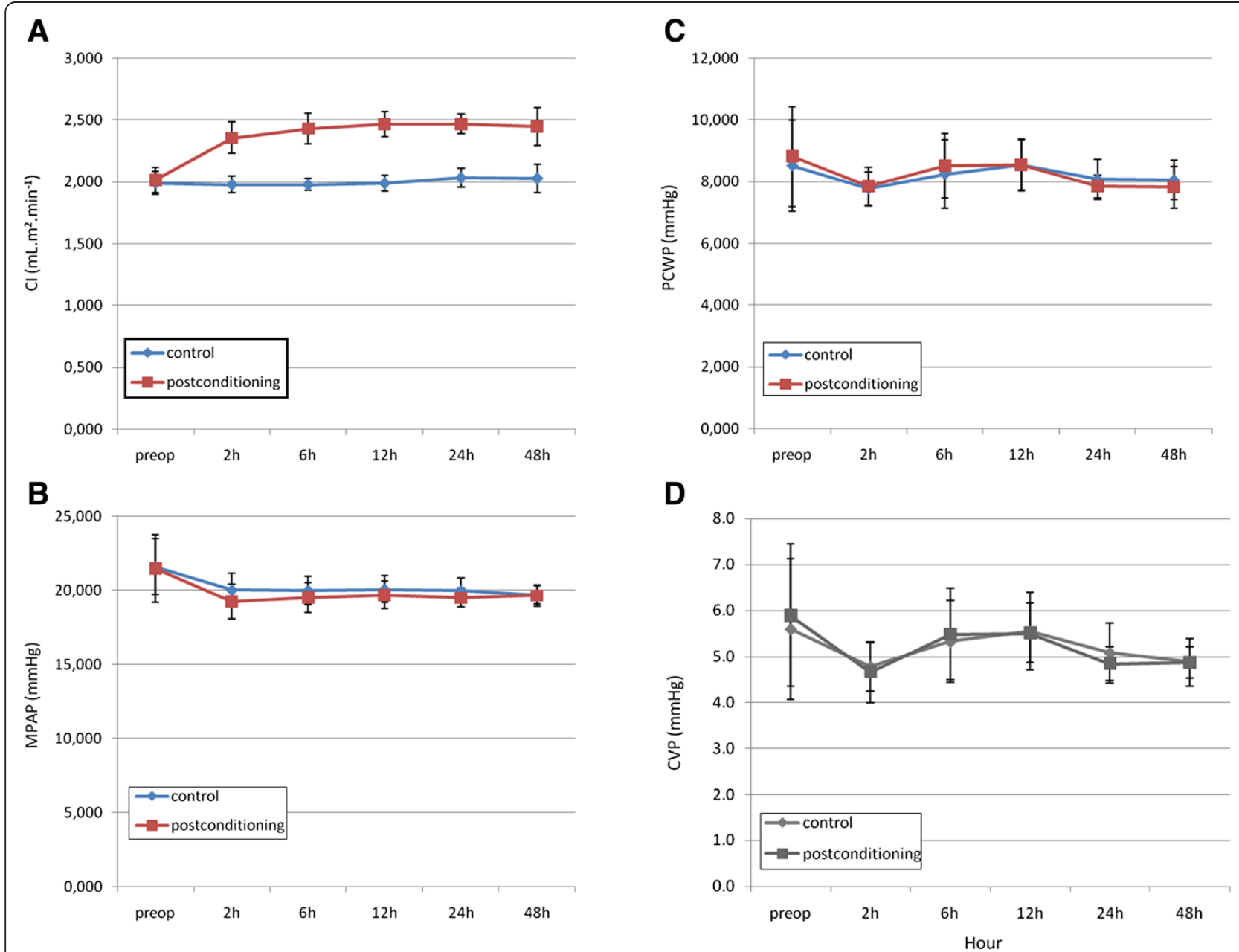

Figure 3 Patients' Hemodynamic Data. A; Time Course of Cardiac Index (Cl). Effect of MMPOC on recovery of $\mathrm{Cl}$ after 2 hours. Preoperative $\mathrm{Cl}$ values were statistically insignificant for the control and postconditioning groups. Postoperative 2 hour point, Cl values for MMPOC group exerted significant difference. After 2 hour point, MMPOC group patients' Cl values showed significant improvement. B; Time Course of Mean Pulmonary Arterial Pressure (MPAP). MPAP values for both groups were statistically insignificant from the beginning of the procedure. C; Time Course of Pulmonary Capillary Wedge Pressure (PCWP). PCWP values for both groups were statistically insignificant from the beginning of the procedure. $\mathbf{D}$; Time Course of Central Venous Pressure (CVP). CVP values for both groups were statistically insignificant from the beginning of the procedure. Blue line: Control, Red line: MMPOC. 
Table 3 Postoperative characteristics

\begin{tabular}{llccc}
\hline & & MMPOC (n= 39) & Control (n=40) & P Value \\
\hline Postoperative inotropes & $\mathrm{n}(\%)$ & $19(48)$ & $32(80)$ & $4(10)$ \\
IABP & $\mathrm{n}(\%)$ & $2(5)$ & $545.6 \pm 97.1$ & 0.004 \\
Bleeding (ml) & Mean \pm SD & $569.2 \pm 120.5$ & $15.6 \pm 3.21$ & 0.214 \\
Mechanical Ventilation (hrs) & Mean \pm SD & $12.0 \pm 3.22$ & $18.8 \pm 3.7$ & 0.818 \\
ICU (hrs) & Mean \pm SD & $15.8 \pm 2.3$ & $5(12.5)$ & 0.000 \\
Postoperative MI & $\mathrm{n}(\%)$ & $3(7.7)$ & $9.1 \pm 2.1$ & 0.712 \\
Hospitalization (days) & Mean \pm SD & $7.5 \pm 1.6$ & $2(5)$ & 0.001 \\
Wound Infection & $\mathrm{n}(\%)$ & $4(10)$ & $5(12.5)$ & $2(5.0)$ \\
Atrial Fibrillation & $\mathrm{n}(\%)$ & $6(15)$ & 0.432 \\
In-hospital mortality & $\mathrm{n}(\%)$ & $0(0)$ & 0.711 \\
\hline
\end{tabular}

$\overline{M M P O C}=$ modified mechanical post-conditioning, $I A B P=$ intra aortic balloon pump, $I C U=$ intensive care unit, $S D=S$ tandard $D e v i a t i o n$.

Nominal variables were assessed by Chi square test or Fisher's Exact test, where applicable. Continuous and ordinal variables were evaluated between two groups by Student's $t$ test and Mann-Whitney $U$ test, respectively.

cardioprotective efficacy of MMPOC in patients undergoing elective coronary revascularization. The current study focused on cardiac biomarker release after CABG surgery. Application of MMPOC prevented the post-bypass increases in TnI, CK and CK-MB.

In our study, to overcome the problem of predicting the volume of at-risk myocardium or collateral perfusion into the ischemic area, we planned to surmount this potentially confounding factor by including only those patients exhibiting proximal occlusion of the LAD artery with or without occlusions of the other coronary arteries as indicated by Darling et al [12]. It can be hypothesized that the better preservation of cardiac function obtained by MMPOC evidenced by data on reduced postoperative TnI release and the reduced need for inotropic support resulted in improved global tissue perfusion with better recovery from surgery as shown by shorter postoperative hospitalization. The mechanisms underlying the benefits of MMPOC are similar to other mechanical POC techniques described before with the advantage of not requiring repetitive aortic cross-clamping.

Post-conditioning as with ischemic preconditioning has the drawback of intermittent cross-clamping which prompted us to modificate the original technique. Unlike pre-conditioning, post-conditioning does not require initiation prior to the ischemic event. This aspect offers several interesting opportunities to the cardiac surgeon. Postconditioning can be used in situations where preconditioning is difficult or impossible to achieve. The cardiac transplant surgeons can also benefit from post-conditioning. The storage time of the cardiac allograft can often exceed the protective effects of early pre-conditioning and therefore, post-conditioning may play an important role for allograft protection following storage and transplantation. Remote post-conditioning has also been demonstrated in an animal model [13]. Kerendi et al. demonstrated in a rodent model that remote post-conditioning using renal artery occlusion reduced infarct size by 50\% [13]. Ischemic post-conditioning requires intermittent cross-clamping of another organ to protect the myocardium which may be associated with morbidity or increased complexity and length of the operative procedure. The optimal postconditioning strategy would be pharmacologic. In this respect a variety of diverse pharmacological postconditioning agents including inhalational anesthetics, Gprotein coupled receptor ligands such as opioids, adenosine and bradykinin, growth factors such as insulin and erythropoietin, natriuretic peptides, adipocytokines, and statins have been linked to the activation of the reperfusion injury salvage kinase pathway, a critical component of this signaling pathway $[14,15]$.

Pharmacological post-conditioning would avoid the adverse consequences associated with intermittent crossclamping and provide a simple method of myocardial protection following all cardiac procedures. A potential drawback of post conditioning is that it offers protection after the ischemic insult has occurred and by then, the myocardial injury may be so extensive that post conditioning is of little benefit [13].

The heightened sensitivity and specificity of $\mathrm{TnI}$ and of CK-MB have rendered such markers ideal in revealing a cardiac injury following an ischemic insult of a different nature. Intraoperative TnI release has a functional significance because it is closely related to ischemic time and reflects delayed recovery of left ventricular function and oxidative metabolism; therefore, its measurement can be used as an indicator of myocardial injury sustained during CABG [16]. A recent study by Luo et. al showed the postoperative release of CK-MB was significantly decreased in POC patients undergoing valve replacement [7]. Moreover, in another study, the same group showed the beneficial effects of POC in a patient subgroup undergoing surgery for tetralogy of Fallot [8]. The major finding of that study is that POC reduced postoperative peak release by $34 \%$ for CK-MB and 
$50 \%$ for TnI, respectively, suggesting that POC reduced myocardial injury. Another study conducted by Kin et. al demonstrated that pharmacologic post conditioning with adenosine administered through an arterial catheter during $\mathrm{CPB}$ produces protective effects against myocardial reperfusion injury in cardiac operations [17]. A possible mechanism of protection, namely, prevention of endothelial ischemia-reperfusion injury by POC was shown by recent studies $[18,19]$.

\section{Conclusion}

We suggest that these cardioprotective effects of MMPOC could have clinical implication in terms of clinical outcome (reduced postoperative length of stay) in CABG operations. Significantly shorter hospital stays will certainly result in lower total hospital costs and nosocomial infections. It can be an adjunct to the other regimes of cardioprotection. Furthermore, our study includes moderately high risk patients with impaired left ventricular ejection faction, medium EuroSCORE and high New York Heart Association (NYHA) status. We assume that improvement in clinical outcome after CABG for patients treated with MMPOC could be demonstrated in a larger study involving patents with worse left ventricular function.

Briefly, our MMPOC technique adjusted after CPB promotes early metabolic recovery of the heart during elective CABG and leads to better myocardial protection and functional recovery.

\section{Abbreviations \\ POC: Post-conditioning; MMPOC: Modified type of mechanical post- conditioning; LAD: Left anterior descending; CPB: Cardiopulmonary bypass; ICU: Intensive care unit; MI: Myocardial infarction; CK-MB: Creatine kinase isoenzyme; CK: Creatine kinase; Tnl: Troponin I; Cl: Cardiac indices; CABG: Coronary artery bypass grafting; CVP: Central venous pressure; MPAP: Mean pulmonary artery pressure; PCWP: Pulmonary capillary wedge pressure; NYHA: New York Heart Association.}

\section{Competing interests}

There is no undisclosed ethical problem or competing interests related to the submitted manuscript.

\section{Authors' contributions \\ SD, MS, DC participated in the design of the study and drafted the manuscript. EO, MBI, ARA, LY, SE, AU participated in performing trial patients and data collection. AHE, DC participated in the statistical analysis. SD, MS conceived of the study, participated in its design and coordination, helped to draft the manuscript and give final approval of the version to be published. All authors read and approved the final manuscript.}

\section{Funding sources}

Ankara University School of Medicine Research Council supported this work.

\section{Acknowledgements}

We sincerely thank Bulent Kaya, Kemalettin Ucanok, Atilla Aral and Umit Ozyurda for their support.

\section{Author details}

${ }^{1}$ Department of Cardiovascular Surgery, Heart Center, Ankara University School of Medicine, Mamak Street, 06340, Dikimevi-Ankara, Turkey. ${ }^{2}$ Stem Cell Institute, Ankara University, Ankara, Turkey. ${ }^{3}$ Department of Biostatistics, Ankara University School of Medicine, Ankara, Turkey.
Received: 25 March 2012 Accepted: 31 July 2012

Published: 9 August 2012

\section{References}

1. Chen Z, Luo H, Zhuang M, Cai L, Su C, Lei Y, Zou J: Effects of ischemic preconditioning on ischemia/reperfusion-induced arrhythmias by upregulatation of connexin 43 expression. J Cardiothorac Surg 2011, 6:80

2. Moens AL, Claeys MJ, Timmermans JP, Vrints CJ: Myocardial ischemia/ reperfusion-injury, a clinical view on a complex pathophysiological process. Int J Cardiol 2005, 100:179-190.

3. Vinten-Johansen J, Zhao ZQ, Jiang R, Zatta AJ: Myocardial protection in reperfusion with postconditioning. Expert Rev Cardiovasc Ther 2005, 3:1035-1045.

4. Zhao ZQ, Vinten-Johansen J: Postconditioning: reduction of reperfusioninduced injury. Cardiovasc Res 2006, 70:200-211

5. PASS. HJNa. Number Cruncher Statistical Systems. Kaysville, Utah; 2001. www. ncss.com.

6. Christakis GT, Fremes SE, Naylor CD, Chen E, Rao V, Goldman BS: Impact of preoperative risk and perioperative morbidity on ICU stay following coronary bypass surgery. Cardiovasc Surg 1996, 4:29-35.

7. Luo W, Li B, Chen R, Huang R, Lin G: Effect of ischemic postconditioning in adult valve replacement. Eur J Cardiothorac Surg 2008, 33:203-208.

8. Luo W, Li B, Lin G, Huang R: Postconditioning in cardiac surgery for tetralogy of Fallot. J Thorac Cardiovasc Surg 2007, 133:1373-1374.

9. Ramzy D, Rao V, Weisel RD: Clinical applicability of preconditioning and postconditioning: the cardiothoracic surgeons view. Cardiovasc Res 2006, 70:174-180

10. Sadat U, Walsh SR, Varty K: Cardioprotection by ischemic postconditioning during surgical procedures. Expert Rev Cardiovasc Ther 2008, 6:999-1006.

11. Luo W, Li B, Lin G, Chen R, Huang R: Does cardioplegia leave room for postconditioning in pediatric cardiac surgery? Cardiol Young 2008 18:282-287.

12. Darling CE, Solari PB, Smith CS, Furman MI, Przyklenk K: Postconditioning' the human heart: multiple balloon inflations during primary angioplasty may confer cardioprotection. Basic Res Cardiol 2007, 102:274-278.

13. Kerendi F, Kin H, Halkos ME, Jiang R, Zatta AJ, Zhao ZQ, Guyton RA, Vinten-Johansen J: Remote postconditioning. Brief renal ischemia and reperfusion applied before coronary artery reperfusion reduces myocardial infarct size via endogenous activation of adenosine receptors. Basic Res Cardiol 2005, 100:404-412.

14. Hausenloy DJ, Yellon DM: Reperfusion injury salvage kinase signaling: taking a RISK for cardioprotection. Heart Fail Rev 2007, 12:217-234.

15. Yang Z, Sun W, Hu K: Molecular mechanism underlying adenosine receptormediated mitochondrial targeting of protein kinase C. Biochim Biophys Acta 2012, 1823:950-958.

16. Koh TW, Hooper J, Kemp M, Ferdinand FD, Gibson DG, Pepper JR: Intraoperative release of troponin $\mathrm{T}$ in coronary venous and arterial blood and its relation to recovery of left ventricular function and oxidative metabolism following coronary artery surgery. Heart 1998, 80:341-348.

17. Kin H, Zatta AJ, Lofye MT, Amerson BS, Halkos ME, Kerendi F, Zhao ZQ, Guyton RA, Headrick JP, Vinten-Johansen J: Postconditioning reduces infarct size via adenosine receptor activation by endogenous adenosine. Cardiovasc Res 2005, 67:124-133.

18. Loukogeorgakis SP, Panagiotidou AT, Yellon DM, Deanfield JE, MacAllister RJ: Postconditioning protects against endothelial ischemia-reperfusion injury in the human forearm. Circulation 2006, 113:1015-1019.

19. Ma X, Zhang X, Li C, Luo M: Effect of postconditioning on coronary blood flow velocity and endothelial function and LV recovery after myocardial infarction. J Interv Cardiol 2006, 19:367-375.

doi:10.1186/1749-8090-7-73

Cite this article as: Durdu et al:: The efficacies of modified mechanical post conditioning on myocardial protection for patients undergoing coronary artery bypass grafting. Journal of Cardiothoracic Surgery 2012 7:73. 